\title{
Automatic analysis of systolic, diastolic and mean blood pressure of continuous measurement before, during and after sleep arousals in polysomnographic overnight recordings
}

\author{
Lerch D. ${ }^{1}$, Orglmeister R. ${ }^{2}$, Penzel T. ${ }^{3}$ \\ ${ }^{1}$ Electronics and Medical Signal Processing, TU Berlin, Berlin, Germany, dennis_lerch@gmx.de \\ ${ }^{2}$ Electronics and Medical Signal Processing, TU Berlin, Berlin, Germany, reinhold.orglmeister@tu-berlin.de \\ ${ }^{3}$ Interdisciplinary Sleep Medicine Center, Charité - Universitätsmedizin, Berlin, Germany, thomas.penzel@charite.de
}

\author{
Structure: 1. Introduction / 2. Methods / 3. Results / 4. Conclusion / 5. References
}

\begin{abstract}
This paper deals with a detailed examination of sleep arousal events and the corresponding changes of systolic, diastolic and mean blood pressure. Arousals are short awakening events during sleep which do not become noticeable for the sleeping person. But the organism increases vital parameters, e.g. the blood pressure. The recreative sleep is disturbed, and the risk factor for cardiovascular diseases rises significantly. Impact on the continuous measured blood pressure for two arousal groups named spontaneous and non spontaneous arousals will be investigated. Polysomnographic recordings of patients suffering from sleep apnoea and a healthy control group will be examined. Using averaged blood pressure curves and a high time resolution, the courses are investigated in more detail than before. The results show an increasing slope a few seconds before and possible pressure minima a few seconds after the beginning of the arousal.
\end{abstract}

\section{Introduction}

Some patients suffer from various sleep disorders. As a consequence they have a disturbed, fragmented and non recreative sleep. Sleep apnoea (breathing interruptions for more than 10 seconds) is a well known sleep disorder. It is assigned as a risk factor for the arterial hypertension [1] and other cardiovascular diseases, such as heart failure and stroke [2]. In the end of an apnoea, the patient unconsciously awakes. In most cases a short sleep arousal event occurs. Other sleep disorders, like periodic leg movement, can also result in short sleep disturbances and arousals.

A cortical arousal is detected in the Electroencephalogram (EEG). It is characterized by an abrupt frequency shift for at least three seconds up to 15 seconds duration and with or without an increasing electromyographic activity [3]. Vital parameters, like blood pressure and heart rate, increase, whereas the sensory threshold decreases [4].

Depending on the cause of stimulus, the arousals can be classified in spontaneous and non spontaneous ones.

Spontaneous arousals can occur 20 times per hour even for healthy persons and do not fragment the sleep. They are also called physiological arousals because of their common occurrence.

But even for a small frequency of five arousals per hour, non spontaneous arousals strongly disturb the recreative sleep [4]. Non spontaneous arousals can be grouped into two subgroups called intrinsic and extrinsic. Intrinsic arousals are organically stimulated, for example by breathing interruptions, leg movement or other disorders. In contrast, the extrinsic arousals are caused by external events. External stimulation covers a wide range from environmental noise, bright light and temperature changes, to disturbances from door clapping or blood pressure measuring.

In previous studies, a correlation between arousals and an increasing maximum blood pressure was shown [5], but a deeper understanding in arousal occurrences and the related activation of the autonomic nervous system is of great interest.

This paper shows the continuous development of the blood pressure curves during different arousals. In particular, temporal correlation between arousal occurrences shown in the EEG and blood pressure reactions will be investigated.

\section{Methods}

\subsection{Data Basis}

In this work, the overnight recordings from the DaphnetProject $^{1}$ are used. Each recording is a cardiorespiratory polysomnography with additional continuous blood pressure measurement. The nights from patients group and healthy persons are scored manually by one medical technician. The patient group consists of 48 night recordings, the healthy group of 43 . The arousal scoring depends on the rules of the American Academy of Sleep

1 Daphnet-Project: Dynamical Analysis of Physiological Networks, EU-Project 2006-2009, Ref. 018474-2, examination of patients suffering from sleep apnoea, periodic leg movement and insomnia. 
Medicine (AASM) [3]. The non invasive continuous blood pressure measurement is performed with the Portapres - System².

\subsection{Data segmentation}

The recordings from patients and healthy subjects are treated separately. Two arousal groups, named spontaneous and ex-/intrinsic, are investigated. The ex-/intrinsic group contains Leg-Movement, Apnoea, Respiratory Effort arousals and arousals stimulated by the blood pressure measurement device. All are classified as non spontaneous ones.

In this study the long-term course of the blood pressure curves is focused and therefore segments of 360 seconds duration in total are analysed. 180 seconds before and 180 seconds after the beginning of an arousal are used. To avoid influences from following or previous arousals, no other arousal occurrences within the 360 seconds segment are allowed. Only arousals which match these interval or gap conditions are considered in the following preprocessing step.

\subsection{Preprocessing}

Due to the measuring technique, the blood pressure curve is corrupted with calibration steps. Furthermore, other artefacts can occur. Therefore, a preprocessing for every segment is necessary. At first, only valid pairs of characteristic blood pressure values, like the systolic and diastolic value, for every heart beat are determined. As a consequence, calibration steps are ignored. If the number of valid blood pressure pairs is below a threshold, the whole curve segment is discarded. This happens if curve segments include long calibration steps and/or other artefacts. Short calibration steps have such a short duration of about one or two heart beats that the missing characteristic blood pressure values can be interpolated with sufficient accuracy.

A minimum threshold of 300 valid blood pressure pairs is chosen, because of the assumption that the minimum heart rate is equal or greater than 50 beats per minute. Figure 1 shows a curve segment during short and long-term calibration processes which does not pass the quality condition.

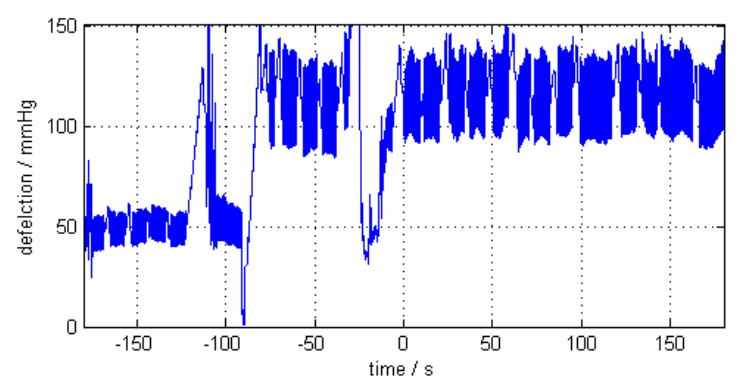

Figure 1 Continuous blood pressure curve segment with calibration steps and other artefacts which is discarded.

2 Non-invasive continuous blood pressure measurement device of the company Finapres Medical Systems BV.
Table 1 gives an overview of usable number of segments in each group for both conditions.

Table 1 Overview of usable arousal segments: a) ex-/intrinsic and b) spontaneous arousals.

\begin{tabular}{|c|c|c|c|c|}
\hline \multirow{2}{*}{$\begin{array}{c}\text { Usable arousal } \\
\text { segments }\end{array}$} & \multicolumn{2}{|c|}{ Patients } & \multicolumn{2}{c|}{ Healthy persons } \\
\cline { 2 - 5 } & $\mathbf{a}$ & $\mathbf{b}$ & $\mathbf{a}$ & $\mathbf{b}$ \\
\hline total & 3574 & 780 & 926 & 1401 \\
\hline $\begin{array}{c}\text { matching gap } \\
\text { condition }\end{array}$ & 368 & 217 & 212 & 453 \\
\hline $\begin{array}{c}\text { matching quality } \\
\text { condition }\end{array}$ & 161 & 103 & 116 & 244 \\
\hline
\end{tabular}

\subsection{Calculating mean averaged curves}

Systolic and diastolic blood pressure values can only be sampled at every heart beat. To reach the same sample rate of the continuous blood pressure signal, intermediate samples will be interpolated. The mean blood pressure curve is the averaged continuous blood pressure during heart cycles and must also be interpolated.

The ensemble average value over all segments and the corresponding standard deviations within the specified groups are determined and plotted for every blood pressure curve. Because of the averaging, individual Gaussian distributed fluctuations in the curve segments are suppressed and the overall trend becomes clearly visible. This procedure is well known as Maximum-Likelihood-Estimation for the true but unknown mean average and standard deviation for a Gaussian distribution. Figure 2 shows one of the resulting plots with the overall trend of the three blood pressure curves (always the three topmost curves) and the resulting standard deviations over the time.

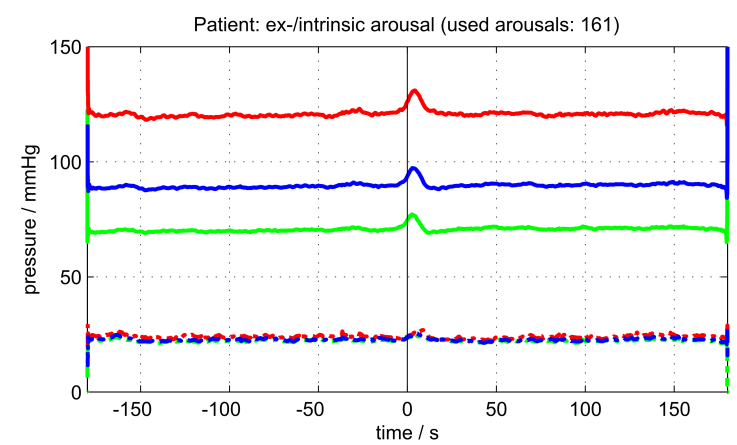

Figure 2 Systolic (red), mean (blue), diastolic averaged pressure (green). Dashed curves are standard deviations.

\section{Results}

For every group four characteristic points in time (in Figure 3 exemplarily marked for the diastolic curve) and the associated blood pressure values are listed in the tables below. Base levels are the values which the curve reaches 
on an average 50 to 150 seconds before and after the arousal beginning.

\subsection{Patient group and ex-/intrinsic arousals}

The resulting curves are shown in Figure 2, and in Table 2 the characteristic points are listed.

The systolic curve has a constant ascent from $121 \mathrm{mmHg}$ to $126 \mathrm{mmHg}-5.8 \mathrm{~s}$ before the arousals' beginning. It ends finally $4.4 \mathrm{~s}$ later in a peak value of about $131 \mathrm{mmHg}$. Afterwards, the curve decreases to the previous base level of about $121 \mathrm{mmHg}$ without having a visible minimum. The diastolic and mean blood pressure curves show a similar behaviour, but weak minima are visible $17 \mathrm{~s}$ and $11 \mathrm{~s}$ later, respectively. All but one standard deviation reach values around $22.6 \mathrm{mmHg}$. Only the systolic standard deviation is a little bit higher. Approximately three seconds after the arousals' beginning, the standard deviations increase by $3 \mathrm{mmHg}$, but these rises do not justify the huge increases of the mean averaged curves. A similar rise occurs at $-165 \mathrm{~s}$ without such a huge rise in the mean averaged curves.

Table 2 Characteristic values from all patients and 161 ex-/intrinsic arousals. "n.v." means no minimum visible.

\begin{tabular}{|c|c|c|c|c|c|c|}
\hline \multirow{2}{*}{$\begin{array}{c}\text { point } \\
\text { in time }\end{array}$} & \multicolumn{2}{|c|}{ systolic } & \multicolumn{2}{c|}{ mean } & \multicolumn{2}{c|}{ diastolic } \\
\cline { 2 - 7 } & $\mathbf{t} / \mathbf{s}$ & $\begin{array}{c}\mathbf{p} / \\
\mathbf{m m H g}\end{array}$ & $\mathbf{t} / \mathbf{s}$ & $\begin{array}{c}\mathbf{p} / \\
\mathbf{m m H g}\end{array}$ & $\mathbf{t} / \mathbf{s}$ & $\begin{array}{c}\mathbf{p} / \\
\mathbf{m m H g}\end{array}$ \\
\hline $\begin{array}{c}\text { slope } \\
\text { rises }\end{array}$ & -5.80 & 121.60 & -5.20 & 90.00 & -5.80 & 71.10 \\
\hline $\begin{array}{c}\text { arousal } \\
\text { onset }\end{array}$ & 0.00 & 126.00 & 0.00 & 93.90 & 0.00 & 75.00 \\
\hline $\begin{array}{c}\text { peak } \\
\text { value }\end{array}$ & 4.40 & 131.00 & 4.00 & 97.00 & 2.80 & 77.10 \\
\hline $\begin{array}{c}\text { after } \\
\text { arousal }\end{array}$ & n.v. & n.v. & 17.00 & 88.00 & 11.40 & 69.10 \\
\hline
\end{tabular}

\subsection{Patient group and spontaneous arousals}

A similar behaviour can be seen in Figure 3 and Table 3. The maximum slope is higher than it was for the ex-/intrinsic arousals, but the standard deviations have slightly higher base levels and variations. However, early increases of the mean averaged curves are clearly visible without huge variations of the standard deviations at the same time. Minima are more obvious in the mean and diastolic blood pressure curve. Spontaneous arousals can occur during different circumstances. As a natural consequence, vital parameters can differ more in comparison to ex-/intrinsic arousal events which occur due to the same stimuli within an arousal group. These effects might be reflected in the standard deviations of the curves.
Table 3 Characteristic values from all patients and 103 spontaneous arousals.

\begin{tabular}{|c|c|c|c|c|c|c|}
\hline \multirow{2}{*}{$\begin{array}{c}\text { point } \\
\text { in time }\end{array}$} & \multicolumn{2}{|c|}{ systolic } & \multicolumn{2}{c|}{ mean } & \multicolumn{2}{c|}{ diastolic } \\
\cline { 2 - 7 } & $\mathbf{t} / \mathbf{s}$ & $\begin{array}{c}\mathbf{p} / \\
\mathbf{m m H g}\end{array}$ & $\mathbf{t} / \mathbf{s}$ & $\begin{array}{c}\mathbf{p} / \\
\mathbf{m m H g}\end{array}$ & $\mathbf{t} / \mathbf{s}$ & $\begin{array}{c}\mathbf{p} / \\
\mathbf{m m H g}\end{array}$ \\
\hline $\begin{array}{c}\text { slope } \\
\text { rises }\end{array}$ & -4.50 & 120.90 & -5.20 & 88.10 & -5.30 & 67.90 \\
\hline $\begin{array}{c}\text { arousal } \\
\text { onset }\end{array}$ & 0.00 & 125.00 & 0.00 & 90.90 & 0.00 & 71.00 \\
\hline $\begin{array}{c}\text { peak } \\
\text { value }\end{array}$ & 5.40 & 133.30 & 5.40 & 98.40 & 5.30 & 76.30 \\
\hline $\begin{array}{c}\text { after } \\
\text { arousal }\end{array}$ & n.v. & n.v. & 14.60 & 83.90 & 14.40 & 64.20 \\
\hline
\end{tabular}

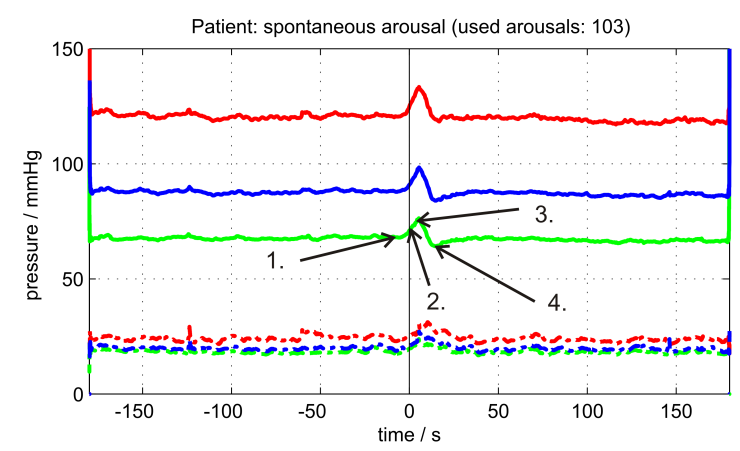

Figure 3 Systolic (red), mean (blue), diastolic averaged pressure (green). Dashed curves are standard deviations. Characteristic points: 1.) slope rises, 2.) arousal onset 3.) peak value 4.) after arousal.

\subsection{Healthy subjects and ex-/intrinsic arousals}

Even if the subjects are in the healthy group, ex-/intrinsic arousals stimulated by Leg-Movement, Respiratory Effort and Apnoea occur. Main differences of the base levels before and after arousal are clearly visible in Figure 4 in comparison with Figure 2. With $120.60 \mathrm{mmHg}$, the base level of the systolic curve from the patient group is approximately $8 \mathrm{mmHg}$ above the base level of the healthy group. This margin can be seen in the other averaged curves too. Table 4 summarises important values.

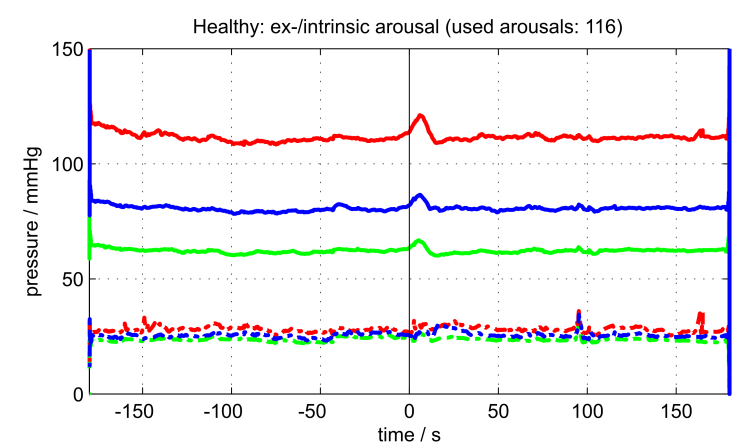

Figure 4 Systolic (red), mean (blue), diastolic averaged pressure (green). Dashed curves are standard deviations. 
Table 4 Characteristic values from all healthy subjects and 116 ex-/intrinsic arousals.

\begin{tabular}{|c|c|c|c|c|c|c|}
\hline \multirow{2}{*}{$\begin{array}{c}\text { point } \\
\text { in time }\end{array}$} & \multicolumn{2}{|c|}{ systolic } & \multicolumn{2}{c|}{ mean } & \multicolumn{2}{c|}{ diastolic } \\
\cline { 2 - 7 } & $\mathbf{t} / \mathbf{s}$ & $\begin{array}{c}\mathbf{p} / \\
\mathbf{m m H g}\end{array}$ & $\mathbf{t} / \mathbf{s}$ & $\begin{array}{c}\mathbf{p} / \\
\mathbf{m m H g}\end{array}$ & $\mathbf{t} / \mathbf{s}$ & $\begin{array}{c}\mathbf{p} / \\
\mathbf{m m H g}\end{array}$ \\
\hline $\begin{array}{c}\text { slope } \\
\text { rises }\end{array}$ & -8.40 & 111.20 & -7.70 & 80.00 & -7.20 & 62.20 \\
\hline $\begin{array}{c}\text { arousal } \\
\text { onset }\end{array}$ & 0.00 & 113.80 & 0.00 & 82.10 & 0.00 & 63.70 \\
\hline $\begin{array}{c}\text { peak } \\
\text { value }\end{array}$ & 5.90 & 121.10 & 5.90 & 86.50 & 5.30 & 66.80 \\
\hline $\begin{array}{c}\text { after } \\
\text { arousal }\end{array}$ & 18.00 & 109.00 & n.v. & n.v. & n.v. & n.v. \\
\hline
\end{tabular}

\subsection{Healthy subjects and spontaneous arousals}

An early increasing slope is not as clearly visible as it is in the previous cases (Figure 5 and Table 5). This effect might be suppressed because of higher standard deviation base levels in comparison with previous curves. In the patients' spontaneous arousal cases, the base levels of the standard deviations are up to $5 \mathrm{mmHg}$ lower. Minima can be seen in all averaged curves, particularly in mean blood pressure and diastolic curves.

Table 5 Characteristic values from all healthy subjects and 244 spontaneous arousals.

\begin{tabular}{|c|c|c|c|c|c|c|}
\hline \multirow{2}{*}{$\begin{array}{c}\text { point } \\
\text { in time }\end{array}$} & \multicolumn{2}{|c|}{ systolic } & \multicolumn{2}{c|}{ mean } & \multicolumn{2}{c|}{ diastolic } \\
\cline { 2 - 7 } & $\mathbf{t} / \mathbf{s}$ & $\begin{array}{c}\mathbf{p} / \\
\mathbf{m m H g}\end{array}$ & $\mathbf{t} / \mathbf{s}$ & $\begin{array}{c}\mathbf{p} / \\
\mathbf{m m H g}\end{array}$ & $\mathbf{t} / \mathbf{s}$ & $\begin{array}{c}\mathbf{p} / \\
\mathbf{m m H g}\end{array}$ \\
\hline $\begin{array}{c}\text { slope } \\
\text { rises }\end{array}$ & -2.00 & 111.60 & -2.00 & 79.20 & -1.80 & 60.20 \\
\hline $\begin{array}{c}\text { arousal } \\
\text { onset }\end{array}$ & 0.00 & 113.30 & 0.00 & 80.70 & 0.00 & 61.30 \\
\hline $\begin{array}{c}\text { peak } \\
\text { value }\end{array}$ & 6.60 & 120.00 & 6.00 & 84.90 & 6.30 & 64.60 \\
\hline $\begin{array}{c}\text { after } \\
\text { arousal }\end{array}$ & 15.00 & 110.50 & 14.80 & 76.80 & 13.90 & 58.10 \\
\hline
\end{tabular}

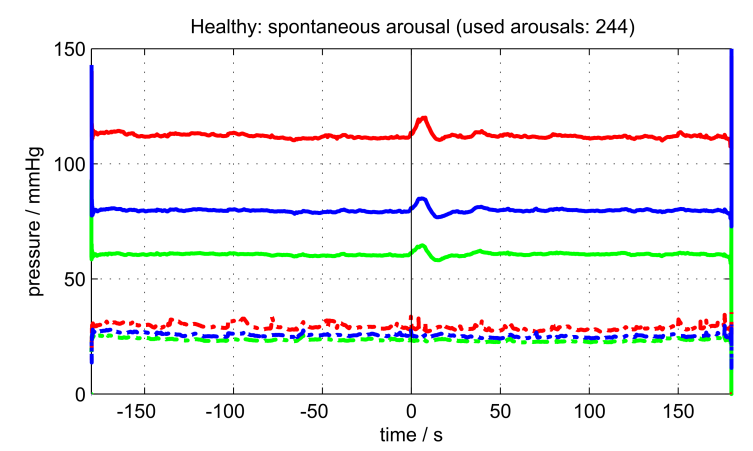

Figure 5 Systolic (red), mean (blue), diastolic averaged pressure (green). Dashed curves are standard deviations.

\section{Conclusion}

The resulting averaged curves show minima approximately $14 \mathrm{~s}$ after arousal beginning. They are clearly visible in the diastolic and mean pressure averaging curves. Furthermore, in the healthy subject group with spontaneous arousals, all three curves have distinct minima. The spontaneous group contains only one arousal type, whereas the ex-/intrinsic group includes three to four different arousal types. This seems to indicate that minima after arousals are depending on the arousal type. Further investigation with split groups will be suitable. In addition, other vital parameters can be examined, e.g. the heart rate, to evaluate the effects.

Patients averaged blood pressure curves increase before the arousal begins no matter which type of arousal occurs, whereas healthy subject's blood pressure indicates only a visible increasing for ex-/intrinsic arousals. In conclusion, it is possible to say that the blood pressure curve shows a rising trend a few seconds before the EEG curve indicates an arousal. Other vital parameters and the associated parameters, e.g. pulse arrival time, should be analysed for similar effects.

In this paper, known effects like a peak blood pressure after an arousal event was verified. A higher base level as it was already mentioned in [6] between patients and healthy persons was recognizable. Advanced examination of the averaged systolic, diastolic and mean blood pressure curves over the time was done. A higher resolution was achieved than in previous examinations which used only characteristic values before, during and after arousal occurrences. As a consequence, an increasing slope before and possible minima after the arousals was found.

\section{$5 \quad$ References}

[1] Peppard, P.; Young, T.; Palta, M.; Skatrud, J.: Prospective study of the association between sleepdisordered breathing and hypertension. N.Engl.J.Med, 2000, 1378-1384

[2] Devulapally, K.; Pongonis, R.; Khayat, R.: OSA: the new cardiovascular disease, Part II: overview of cardiovascular diseases associated with obstructive sleep apnea, Heart Failure Reviews, Volume 14, Number 3, 2009, 155-164

[3] Iber, C.; et al.: The AASM Manual for the scoring of sleep and associated events. Westchester, Illinois: $1^{\text {st }}$ ed. American Academy of Sleep Medicine, 2007

[4] Peter, H.; Penzel, T.; Jörg Hermann, P. (eds.): Enzyklopädie der Schlafmedizin, 2007

[5] Klaus, W.: Definition und Bedeutung autonomer Arousal bei Patienten mit obstruktiver Schlafapnoe und bei Schlafgesunden. Phd. thesis, Charité Universitätsmedizin, Berlin, 2011

[6] Stradling, J.; Barbour, C.; Glennon, J.; Langford, B.; Crosby, J.: Which aspects of breathing during sleep influence the overnight fall of blood pressure in a community population? Thorax, 2000;55, 393-398 